\title{
Trapped surfaces and spherical closed cosmologies.
}

\author{
Edward Malec ${ }^{+}$and Niall Ó Murchadha* \\ + Instytut Fizyki, Uniwersytet Jagielloński 30-064 Kraków, Reymonta 4, Poland \\ * Physics Department, University College, Cork, Ireland \\ PACS numbers: 04.20., 98.80., 95.30., 97.60.
}

\begin{abstract}
.
This article gives necessary and sufficient conditions for the formation of trapped surfaces in spherically symmetric initial data defined on a closed manifold. Such trapped surfaces surround a region in which there occurs an enhancement of matter over the average. The conditions are posed directly in terms of physical variables and show that what one needs is a relatively large amount of excess matter confined to a small volume. The expansion of the universe and an outward flow of matter oppose the formation of trapped surfaces; an inward flow of matter helps. The model can be regarded as a FriedmannLemaittre-Walker cosmology with localized spherical inhomogeneities. We show that the total excess mass cannot be too large.
\end{abstract}




\section{Introduction}

We intend to study the geometry of initial data for the Einstein equations coupled with a matter field so as to investigate the presence (or absence) of trapped surfaces. A trapped surface is a 2-surface which exists at a particular instant of time and has the property that all the outgoing light-rays from it converge $[1,2]$. One expects that outgoing light-rays diverge, so one can immediately deduce that the gravitational field in the vicinity of a trapped surface must be very strong so as to prevent the light-rays from expanding. The presence of a trapped surface indicates that the spacetime is undergoing gravitational collapse. One of the singularity theorems of general relativity states that if one has a trapped surface (and if the enclosed volume is finite) then there must be a singularity to the future (e.g., [2]). If one accepts Cosmic Censorship, that singularities are hidden, then the presence of a trapped surface is a sign that a black hole is in the process of forming.

Initial data for the gravitational field consists of four objects $\left[g_{a b}, K^{a b}, \rho\right.$ and $\left.J^{a}\right]$, where $g_{a b}$ is the three-dimensional positive definite metric of a 3 -hypersurface $\Sigma ; \Sigma$ is to be regarded as a spacelike slice through the 4-manifold. $K^{a b}$ is a symmetric 3 -tensor which is the extrinsic curvature of $\Sigma$ as an embedded surface, $\rho$ is the energy density and $J^{a}$ is the momentum density of the matter. These data are not independent, they must satisfy the constraint equations

$$
\begin{gathered}
{ }^{(3)} R[g]-K_{a b} K^{a b}+\left(K_{a}^{a}\right)^{2}=16 \pi \rho, \\
\nabla_{a} K^{a b}-\nabla^{b} K^{a}{ }_{a}=-8 \pi J^{b},
\end{gathered}
$$

where ${ }^{(3)} R[g]$ is the scalar curvature of $\Sigma$ and $\nabla$ is the 3-covariant derivative compatible with $g_{a b}$. 
The trace of the extrinsic curvature $\left(\operatorname{tr} K=g_{a b} K^{a b}\right)$ is equal to the (positive) time rate of change of the three-volume, $\frac{d}{d t}\left(d^{3} V\right)=\operatorname{tr} K d V$. It is very common to place conditions on trK so as to select a preferential slicing ("extrinsic time"). In asymptotically flat space-times a standard choice is the maximal slicing condition, $\operatorname{trK}=0$. In cosmological models(e.g. [3]), the analogous condition is to choose $\operatorname{trK}=$ constant.

A trapped surface is defined as a compact two-dimensional (smooth) spacelike surface S having the property that the expansion $\theta$ of outgoing future-directed null geodesics which are orthogonal to $\mathrm{S}$ is everywhere negative on $\mathrm{S}$. If $\mathrm{S}$ is regarded as a submanifold of $\Sigma$, then $\theta$ can be expressed in terms of $g_{a b}$ and $K^{a b}$ by

$$
\theta=\nabla_{a} n^{a}-K_{a b} n^{a} n^{b}+g_{a b} K^{a b},
$$

where $n^{a}$ is the outward unit normal to $\mathrm{S}$ in $\Sigma$.

We have recently derived simple necessary and sufficient conditions for the appearance of trapped surfaces in asymptotically flat initial data sets [4] and in open universes [5], with the additional assumption that the initial data are spherically symmetric. In this article we will derive equivalent necessary and sufficient conditions for trapped surfaces in a closed universe. We continue to demand spherical symmetry and we impose the condition that the data satisfy $\operatorname{trK}=$ constant. The problem we wish to consider looks like a spherical lump superimposed on a standard Friedmann-Lemaître-Walker (FLW hereafter) background, in the fact that we assume that the density becomes constant outside some finite subset and the matter current vanishes outside the same subset. We address also the situation where a closed universe is filled with many spherically symmetric bumps that have a small domain of influence so that in between them geometry coincides with the homogeneous 
and isotropic FLW geometry .

The standard time-slice through a closed FLW cosmology is defined by a threemanifold which is just the standard round three-sphere, i.e., the line-element can be written as

$$
d s^{2}=a^{2}\left[d r^{2}+\sin ^{2} r d \Omega^{2}\right]
$$

where $a$ is a constant; $K^{a b}$ is pure trace, i.e., $K^{a b}=H g^{a b}$, where $H$ is a constant, the Hubble constant; the energy density is a constant, $\rho_{0}$, and the matter current density is zero. The scalar curvature of the manifold is given by

$$
{ }^{(3)} R=\frac{6}{a^{2}},
$$

so therefore the Hamiltonian constraint reduces to

$$
\frac{6}{a^{2}}+6 H^{2}=16 \pi \rho_{0}
$$

The momentum constraint is trivial.

The normal $n^{a}$ to the two-surfaces of constant radius is given by $n^{a}=\left(a^{-1}, 0,0\right)$ and its divergence is given by

$$
\nabla_{a} n^{a}=\left(a \sin ^{2} r\right)^{-1} \frac{d \sin ^{2} r}{d r}=\frac{2}{a} \cot r
$$

Therefore the expansion $\theta$ of such surfaces is given by

$$
\theta=\frac{2 \cot r+2 a H}{a}
$$

If the universe is expanding $(H>0)$, then trapped surfaces can only occur on the 'other' side of the equator, i.e., $r>\frac{\pi}{2}$, where $\cot r$ is negative. Since $\cot r$ becomes unboundedly 
large and negative, we will always find trapped surfaces. These trapped surfaces indicate the existence of the final 'big crunch'. This property should hold for any spherically symmetric universe and so finding trapped surfaces near the 'south pole' is not very interesting. Thus we will be interested in finding trapped surfaces in the 'northern' hemisphere, far away from the 'south pole'. These, we expect, should indicate the onset of some local gravitational collapse, superimposed on the overall expansion and subsequent collapse of the whole universe.

The initial data $\left[g_{a b}, K^{a b}, \rho, J^{a}\right]$ we wish to consider are defined on a three-manifold $\Sigma$, which has topology $S^{3}$. We assume $\operatorname{tr} K=g_{a b} K^{a b}=3 H=$ constant holds everywhere on the 3-manifold. We further assume that the metric, the extrinsic curvature, $\rho$, and $J^{a}$ are spherically symmetric. Because of the spherical symmetry we know that the three-metric is conformally flat and so can write the line-element in isotropic coordinates as

$$
d s^{2}=a^{2} \phi^{4}\left[d r^{2}+\sin ^{2} r d \Omega^{2}\right],
$$

where $a$ is a positive constant and $\phi$ is a positive conformal factor that depends only on $r$. We assume that $\rho$ and $J^{a}$ are arbitrary (other than satisfying some positivity conditions one may wish to impose). We do not impose any equation of state on the matter. We need not do so as we restrict attention to a single instant of time, we make no use of the Einstein evolution equations. We further assume that $\rho=\rho_{0}$ (a constant) and $J^{a}=0$ outside some subregion of compact support. We choose the constant $a$ in eqn.(9) to satisfy

$$
\frac{6}{a^{2}}+6 H^{2}=16 \pi \rho_{0}
$$

so as emphasise the link with the FLW cosmology. 


\section{The extrinsic curvature in spherically symmetric closed universes}

We assume that the extrinsic curvature is spherically symmetric. This means that the general form of $K^{a b}$ is

$$
K^{a b}=K(r) n^{a} n^{b}+b(r) g^{a b},
$$

where $n^{a}$ is the unit vector in the radial direction, and $K(r)$ and $b(r)$ are two scalar functions. Since we know $\operatorname{trK}=3 \mathrm{H}$, we can rewrite (11) as

$$
K^{a b}=H g^{a b}+K(r)\left[n^{a} n^{b}-\frac{g^{a b}}{3}\right] .
$$

The momentum constraint, Eq. (2), reads

$$
\frac{2}{3 a^{2} \phi^{4}}\left[K^{\prime}+\left(6 \frac{\phi^{\prime}}{\phi}+3 \frac{\cos r}{\sin r}\right) K\right]=-\frac{8 \pi J}{a \phi^{2}},
$$

where we assume $J^{a}=J n^{a}$, with $n^{a}=\left(a^{-1} \phi^{-2}, 0,0\right)$ as the unit vector in the radial direction, $J=J(r)$ is a scalar and the primes represent derivatives with respect to $r$. Eq. (13) can be slightly simplified to

$$
K^{\prime}+\left(6 \frac{\phi^{\prime}}{\phi}+3 \frac{\cos r}{\sin r}\right) K=-12 \pi a \phi^{2} J
$$

Now let us make our standard assumption, that $\mathrm{J}$ is zero outside some finite subset. The momentum constraint (Eq. (14)) reduces to

$$
K^{\prime}+\left(6 \frac{\phi^{\prime}}{\phi}+3 \frac{\cos r}{\sin r}\right) K=0
$$

in the exterior region. It is easy to solve this equation to give

$$
K=C\left(\phi^{6} \sin ^{3} r\right)^{-1},
$$


where $C$ is some constant. This is a well-known result, in disguise. $K$ generates a divergence-free, tracefree (TT) tensor. The spherically symmetric closed manifold is conformally flat, and TT tensors are conformally covariant. There is a unique spherically symmetric TT tensor on flat space, and the tensor $K\left(n^{a} n^{b}-g^{a b} / 3\right)$, with $K$ given by Eq. (16), is exactly this tensor, transformed in the correct way.

The constant $C$ is essentially the integral of the matter current. Eq. (14) can be rewritten as

$$
\left[K \phi^{6} \sin ^{3} r\right]^{\prime}=-12 \pi a \phi^{8} \sin ^{3} r J
$$

This can be integrated from $r=0$ to $r=R$ to give

$$
K(R)=-12 \pi a\left[\phi^{6}(R) \sin ^{3} R\right]^{-1} \int_{0}^{R} \phi^{8} \sin ^{3} r J d r
$$

One important consequence of $(18)$ is that if the matter is at rest, i.e., $J \equiv 0$, then we can immediately deduce that $K \equiv 0$ and the extrinsic curvature is pure trace. Another consequence is that is that if we have a globally spherically symmetric model with the matter current confined to a region near the north pole, the integral in Eq. (18) when we let $\mathrm{R}$ approach $\pi$ must go to zero. Otherwise, the extrinsic curvature term in the Hamiltonian constraint will have a term that diverges like $\sin ^{-6} r$ as $\mathrm{r}$ approaches $\pi$. In other words, if $J$ vanishes outside a finite region (and we have global spherical symmetry) so also will $K$.

\section{The Hamiltonian constraint in spherically symmetric closed universes}

We now can put Eqs. (9) and (12) together and evaluate the Hamiltonian constraint.

We can write the scalar curvature as ${ }^{(3)} R=6 a^{-2} \phi^{-4}-8 \phi^{-5} \nabla^{2} \phi$, where the laplacian is 
with respect to the background metric (Eq. (4)). We also have that $(\operatorname{tr} K)^{2}-K^{a b} K_{a b}=$ $6 H^{2}-\frac{2}{3} K^{2}$. Thus the hamiltonian constraint, Eq. (1), reads

$$
\frac{6}{a^{2}} \phi^{-4}-8 \phi^{-5} \nabla^{2} \phi+6 H^{2}-\frac{2}{3} K^{2}=16 \pi \rho .
$$

Rearranging, and using Eq. (10), we get

$$
\nabla^{2} \phi=\frac{3}{4 a^{2}}\left(\phi-\phi^{5}\right)-\left[\frac{1}{12} K^{2}+2 \pi\left(\rho-\rho_{0}\right)\right] \phi^{5} .
$$

This is our key equation and much of our effort will be devoted to analysing it.

The other important quantity we need to consider is the expansion $\theta$ (Eq. (3)) of the surfaces of constant $r$. The normal to such surfaces is given by $n^{a}=\left(a^{-1} \phi^{-2}, 0,0\right)$, and $\nabla_{a} n^{a}$ is given by

$$
\nabla_{a} n^{a}=\left(a \phi^{6} \sin ^{2} r\right)^{-1}\left(\phi^{4} \sin ^{2} r\right)^{\prime}=2\left(a \phi^{3} \sin r\right)^{-1}\left(2 \phi^{\prime} \sin r+\phi \cos r\right) .
$$

On using Eq. (12) we get $-K_{a b} n^{a} n^{b}+g_{a b} K^{a b}=2 H-\frac{2}{3} K$. Hence we get (from (3))

$$
\theta=2\left(a \phi^{3} \sin r\right)^{-1}\left(2 \phi^{\prime} \sin r+\phi \cos r\right)+2 H-\frac{2}{3} K .
$$

The aim of this article is to take the expression (22) for the expansivity in terms of the 'potentials' $\phi$ and K, and use equations (18) and (20) to replace these quantities with $\rho$ and $\vec{J}$, more physical objects. Two other spherically symmetric cases have already been successfully dealt with in this fashion, the case where the three-geometry is asymptotically flat and the case where the system asymptotically approaches a flat Friedmann cosmology $[4,5]$. 


\section{A sufficient condition for trapped surfaces when $\mathbf{J}=0$.}

We assume that we are given spherically symmetric initial data $\left[g_{a b}, K^{a b}, \rho, \vec{J}\right]$ (which may be confined to a finite region). We assume that the trace of the extrinsic curvature is constant. We further specialize by assuming that the matter-current is instantaneously zero. As we have shown in Section II, this allows us to conclude that the extrinsic curvature is pure trace, so that the function $K(r) \equiv 0$. We also assume that $\rho$ assumes a constant value $\rho_{0}$ outside a region of compact support. The Hamiltonian constraint now reduces to a simplified version of Eq. (20)

$$
\nabla^{2} \phi=\frac{3}{4 a^{2}}\left(\phi-\phi^{5}\right)-2 \pi \Delta \rho \phi^{5},
$$

where $\Delta \rho=\rho-\rho_{0}$.

We now wish to get some relationship between our sources (in this case $\Delta \rho$ ) and the expansion $\theta$ of any given spherical surface, with coordinate radius $r=R_{0}$ in the northern hemisphere. The trick is to take Eq. (23), multiply it by $\phi$ and integrate it in the background metric, over the volume $\mathrm{V}$ enclosed by the surface at $R_{0}$. This gives

$$
\int_{V} \phi \nabla^{2} \phi d V=\frac{3}{4 a^{2}} \int_{V}\left(\phi^{2}-\phi^{6}\right) d V-2 \pi \int_{V} \Delta \rho \phi^{6} d V .
$$

The left-hand-side of (24) can now be written as

$$
\begin{aligned}
\int_{V} \phi \nabla^{2} \phi d V & =\oint_{R_{0}} \phi \nabla_{a} \phi \cdot d S^{a}-\int_{V}(\nabla \phi)^{2} d V \\
& =\left.4 \pi a \phi \phi^{\prime} \sin ^{2} r\right|_{R_{0}}-4 \pi a \int_{0}^{R_{0}}\left(\phi^{\prime}\right)^{2} \sin ^{2} r d r .
\end{aligned}
$$

Now consider the expression (22) for $\theta$, dropping the term in K. This can be rearranged to give

$$
\left(\pi a^{2} \phi^{4} \sin ^{2} r\right)(\theta-2 H)=4 \pi a \phi \phi^{\prime} \sin ^{2} r+2 \pi a \phi^{2} \sin r \cos r .
$$


We should recognise that $4 \pi a^{2} \phi^{4} \sin ^{2} r=A$ is the proper area of a sphere of coordinate radius $\mathrm{r}$ in the physical space. We now merge (26) with (25) to give

$$
\int_{V} \phi \nabla^{2} \phi d V=\frac{A}{4}\left(\left.\theta\right|_{R_{0}}-2 H\right)-4 \pi a \int_{0}^{R_{0}}\left(\phi^{\prime}\right)^{2} \sin ^{2} r d r-2 \pi a \phi^{2} \sin R_{0} \cos R_{0} .
$$

We can simplify the right-hand-side of (24) when we recognise that

$$
\int_{V} \phi^{6} d V=V
$$

where $\mathrm{V}$ is the proper volume enclosed by the surface, and

$$
\int_{V} \Delta \rho \phi^{6} d V=\Delta M
$$

where $\Delta M$ is the mass excess as measured in the physical space. We now combine (24) and (27) to give

$$
\left.\frac{A}{4} \theta\right|_{R_{0}}=\pi a \int_{0}^{R_{0}}\left[3 \phi^{2} \sin ^{2} r+4\left(\phi^{\prime}\right)^{2} \sin ^{2} r+2\left(\phi^{2} \sin r \cos r\right)^{\prime}\right] d r+\frac{A H}{2}-\frac{3 V}{4 a^{2}}-2 \pi \Delta M .
$$

Consider the integrand of the integral on the right-hand-side of (30). It is

$$
\begin{aligned}
I & =\phi^{2} \sin ^{2} r+4\left(\phi^{\prime}\right)^{2} \sin ^{2} r+4 \phi \phi^{\prime} \sin r \cos r+2 \phi^{2} \cos ^{2} r \\
& =2 \phi^{2}-\phi^{2} \sin ^{2} r+4 \phi^{\prime} \sin r\left(\phi^{\prime} \sin r+\phi \cos r\right) .
\end{aligned}
$$

Let us now make two (as yet unjustified) assumptions. We will assume

$$
\phi^{\prime}<0 \text { and } \phi^{\prime} \sin r+\phi \cos r>0
$$

on the support of the matter.In later sections we will show that these conditions follow naturally from the Hamiltonian constraint (20), if we assume that the excess mass $\Delta \rho$ is 
positive. Almost identical conditions have been derived and used in [4] and [5]. We now can deduce

$$
I<2 \phi^{2} .
$$

Thus we obtain the inequality we want

$$
\left.\frac{A}{4} \theta\right|_{R_{0}}<2 \pi a \int_{0}^{R_{0}} \phi^{2} d r+\frac{A H}{2}-\frac{3 V}{4 a^{2}}-2 \pi \Delta M .
$$

We can identify $a \int_{0}^{R_{0}} \phi^{2} d r=L$ as the proper radius of the sphere of coordinate radius $R_{0}$. Thus we can write inequality (35) as

$$
\left.\frac{A}{8 \pi} \theta\right|_{R_{0}}<L+\frac{A H}{4 \pi}-\frac{3 V}{8 \pi a^{2}}-\Delta M
$$

We have proven the following:

Lemma 1:Assuming conditions (33), if we can find in our physical data a spherical surface satisfying

$$
\Delta M>L+\frac{A H}{4 \pi}-\frac{3 V}{8 \pi a^{2}}
$$

then that surface is trapped.

If we were interested in minimal surfaces rather than in trapped surfaces we can prove:

Lemma 2: Under the conditions stated in this section, if we can find in our physical data a spherical surface satisfying

$$
\frac{1}{16 \pi} \Delta^{(3)} R>L-\frac{3 V}{8 \pi a^{2}}
$$

where $\Delta^{(3)} R$ is the excess integrated scalar curvature, then the manifold must contain a minimal surface. 


\section{A necessary condition for trapped surfaces when $\mathbf{J}=\mathbf{0}$}

It is possible to find a necessary condition for trapped surfaces under much weaker conditions. We need make no assumptions such as (33). Let us return to equation (30) and consider again the integrand (31)

$$
\begin{aligned}
I & =\phi^{2} \sin ^{2} r+4\left(\phi^{\prime}\right)^{2} \sin ^{2} r+4 \phi \phi^{\prime} \sin r \cos r+2 \phi^{2} \cos ^{2} r \\
& =\phi^{2}+\left[\phi \cos r+2 \phi^{\prime} \sin r\right]^{2} \\
& >\phi^{2}
\end{aligned}
$$

It is interesting to note that we do not impose any conditions on $\phi$ or $\phi^{\prime}$ to derive this inequality. When (40) is substituted back into (30) we get

$$
\left.\frac{A}{4} \theta\right|_{R_{0}}>\pi a \int_{0}^{R_{0}} \phi^{2} d r+\frac{A H}{2}-\frac{3 V}{4 a^{2}}-2 \pi \Delta M
$$

Again we use $\int_{0}^{R_{0}} a \phi^{2} d r=L$, the proper radius of the sphere to simplify (41) to

$$
\left.\frac{A}{8 \pi} \theta\right|_{R_{0}}>\frac{L}{2}+\frac{A H}{4 \pi}-\frac{3 V}{8 \pi a^{2}}-\Delta M
$$

Thus we have

Theorem I: Any spherical surface in the physical data satisfying

$$
\Delta M<\frac{L}{2}+\frac{A H}{4 \pi}-\frac{3 V}{8 \pi a^{2}}
$$

cannot be trapped.

Let us stress again that this inequality is valid for any spherical surface in the whole universe. As a trivial application, it is compatible with the fact that all the surfaces near 
the south pole are trapped, even with no extra mass. As we approach the south pole, in the standard Friedmann model, we get $L \sim a \pi, V \sim 2 \pi^{2} a^{3}$ and $A \sim 0$. Thus the right-hand-side of (43) approaches $-\pi a / 4$.

\section{The effect of a matter current on trapped surface formation}

In Sections IV and V we have dealt with the situation where the matter current was at rest. In this section we will consider the effects of nonzero $\vec{J}$. We will continue to assume that both the excess matter and the current density are confined to the northern hemisphere and that the trace of the extrinsic curvature is a constant. The hamiltonian constraint has the general form as given by (20)

$$
\nabla^{2} \phi=\frac{3}{4 a^{2}}\left(\phi-\phi^{5}\right)-\left[\frac{1}{12} K^{2}+2 \pi \Delta \rho\right] \phi^{5}
$$

and we see that the effect of the nonzero current is just to add another positive term to $\Delta \rho$. We need to derive an equation similar to (30), but taking into account the extra terms that arise in both the definition of the expansion and in the Hamiltonian constraint. Before we do so, it is useful to multiply the momentum constraint by the unit radial vector and integrate it over a spherical volume in the physical space. We assume that the extrinsic curvature has the form given by (12)

$$
K^{a b}=H g^{a b}+K(r)\left[n^{a} n^{b}-\frac{g^{a b}}{3}\right]
$$

where $\mathrm{H}$ is a constant. We thus wish to consider

$$
\int_{V}\left(n_{a} \nabla_{b} K^{a b}-n^{a} \nabla_{a} K_{b}^{b}\right) d V=-8 \pi \int_{V} n_{a} J^{a} d V
$$


The term in $\mathrm{H}$ contributes nothing to the integral so we can ignore it. Thus we can replace $K^{a b}$ in the integral with $k^{a b}=K(r)\left[n^{a} n^{b}-g^{a b} / 3\right]$. The second term on the left in (46) is zero as well. Now we integrate by parts the remaining term on the left to give

$$
\left.A n_{a} n_{b} k^{a b}\right|_{R_{0}}-\int_{V} k^{a b} \nabla_{a} n_{b} d V=-8 \pi \int_{V} n_{a} J^{a} d V
$$

This can be further simplified to read

$$
\left.\frac{2}{3} K A\right|_{R_{0}}+\frac{1}{3} \int_{V} K \nabla_{a} n^{a} d V=-8 \pi \int_{V} n_{a} J^{a} d V
$$

Substituting from Eq. (21) finally gives

$$
\left.\frac{2}{3} K A\right|_{R_{0}}+\frac{8 \pi a}{3} \int_{0}^{R_{0}} K a \phi^{3} \sin r\left(2 \phi^{\prime} \sin r+\phi \cos r\right) d r=-8 \pi \int_{V} n_{a} J^{a} d V .
$$

Let us now return to the Hamiltonian constraint. Repeating the manipulation of Section $\mathrm{V}$ leads to an equation analogous to (30)

$$
\left.\frac{A}{4} \theta\right|_{R_{0}}=\pi a \int_{0}^{R_{0}}\left[I-\frac{1}{3}\left(K a \phi^{3} \sin r\right)^{2}\right] d r+\frac{A H}{2}-\frac{A K}{6}-\frac{3 V}{4 a^{2}}-2 \pi \Delta M,
$$

where I is exactly the integrand given in (31). Let us eliminate the $A K$ term from (50) by subtracting $1 / 4$ of (49) from it. To simplify the notation somewhat, we will replace $K a \phi^{3} \sin r$ by $k$ and divide by $2 \pi$ to give

$$
\left.\frac{A}{8 \pi} \theta\right|_{R_{0}}=\frac{a}{2} \int_{0}^{R_{0}}\left[I-\frac{1}{3} k^{2}+\frac{2}{3} k\left(2 \phi^{\prime} \sin r+\phi \cos r\right)\right] d r+\frac{A H}{4 \pi}-\frac{3 V}{8 \pi a^{2}}-(\Delta M-\Delta J),
$$

where we define $\Delta J=\int_{V} n_{a} J^{a} d V$.

We can write the total integrand in (51) as

$I^{\prime}=\phi^{2}\left(\sin ^{2} r+7 / 3 \cos ^{2} r\right)+8 \phi^{\prime} \sin r\left(2 \phi^{\prime} \sin r+\phi \cos r-k / 3\right)-\frac{1}{3}\left(k-6 \phi^{\prime} \sin r-\phi \cos r\right)^{2}$. 
The first term in the integrand $I^{\prime}$ is easy to handle. It can be written as

$$
I_{1}=\phi^{2}\left(7 / 3-4 / 3 \sin ^{2} r\right)<\frac{7}{3} \phi^{2},
$$

and the integral of this term is $<7 L / 6$. The middle term can be written, on using (22) as

$$
\begin{aligned}
I_{2} & =8 a \phi^{3} \phi^{\prime} \sin ^{2} r(\theta / 2-H) \\
& =4 a \theta \phi^{\prime} \phi^{3} \sin ^{2} r-8 H a \phi^{3} \phi^{\prime} \sin ^{2} r \\
& =4 a \theta \phi^{\prime} \phi^{3} \sin ^{2} r-2 H a\left(\phi^{4} \sin ^{2} r\right)^{\prime}+4 H a \phi^{4} \sin r \cos r .
\end{aligned}
$$

If we have no trapped surfaces in the interior, we have that $\theta>0$ and therefore the first term in (56) is obviously negative, on using our standard inequality $(33)$, i.e., $\phi^{\prime}<0$. The second term integrates to give $-A H / 4 \pi$, which cancels the equivalent term in (51). The only term that we need to consider carefully is the third term in (56). The integral of this term can be estimated as follows. Let us define

$$
\Gamma=2 H a^{2} \int_{0}^{R} \phi^{4} \sin r \cos r d r
$$

We can show that $\Gamma<H L^{2}$. To obtain this we need to use $r^{-1} \sin r \cos r \leq 1$. This allows us to write

$$
\Gamma<2 H \int_{0}^{R}\left(a r \phi^{2}\right)\left(a \phi^{2} d r\right)
$$

If we assume $\phi^{\prime}<0$ we have that $\phi$ monotonically decreases so we know that, at any point, $a \phi^{2} r<L$, and, of course, $a \phi^{2} d r=d L$. This immediately gives us the desired result.

Let us now return to Eq. (52) and assume that there is no trapped surface inside the radius $R_{0}$ we are considering. We can now write

$$
\left.\frac{A}{8 \pi} \theta\right|_{R_{0}}<\frac{7}{6} L+H L^{2}-\frac{3 V}{8 \pi a^{2}}-(\Delta M-\Delta J) .
$$


This now will give us our desired result:

Lemma 3: Assuming $\phi^{\prime}<0$, if we obtain a surface for which

$$
\Delta M-\Delta J>\frac{7}{6} L+H L^{2}-\frac{3 V}{8 \pi a^{2}}
$$

either the surface is itself trapped or the interior contains trapped surfaces.

Another estimate can be derived if we are willing to use both conditions in (33). We can write the total integrand $I^{\prime}$ in (51) in a different way. We get

$$
I^{\prime}=\phi^{2}\left(\sin ^{2} r+7 / 3 \cos ^{2} r\right)+\frac{16}{3} \phi^{\prime} \sin r\left(\phi^{\prime} \sin r+\phi \cos r\right)-\frac{1}{3}\left(k-2 \phi^{\prime} \sin r-\phi \cos r\right)^{2} .
$$

If we accept (33) the middle term is negative, the last term is obviously negative, and, as in (53), the first term is less than $\frac{7}{3} \phi^{2}$. This means that we can replace (59) with

$$
\left.\frac{A}{8 \pi} \theta\right|_{R_{0}}<\frac{7}{6} L+\frac{A H}{4 \pi}-\frac{3 V}{8 \pi a^{2}}-(\Delta M-\Delta J) .
$$

This gives us

Lemma 4: Assuming conditions (33), if we obtain a surface for which

$$
\Delta M-\Delta J>\frac{7}{6} L+\frac{A H}{4 \pi}-\frac{3 V}{8 \pi a^{2}}
$$

the surface is trapped.

Note: Lemma 4 is usually better than Lemma 3 because if we assume $\phi^{\prime}<0$ we immediately get $A<4 \pi L^{2}$. 


\section{The total excess mass must be bounded}

There are many inequalities that one can derive using these techniques, but one, in particular, is enlightening i $\mathrm{n}$ that it shows that there are very strict bounds on the total amount of excess mass that can be placed in finite region, independent of whether this matter is inside a horizon or not. Let us return to eqns.(23) and (24) but assume that $J$ is nonzero. These can be written as

$$
\int K^{2} d V+2 \pi \Delta M=-\left.4 \pi a \phi \phi^{\prime} \sin ^{2} r\right|_{R_{0}}+\pi a \int_{0}^{R_{0}}\left(3 \phi^{2} \sin ^{2} r+4\left(\phi^{\prime}\right)^{2} \sin ^{2} r\right) d r-\frac{3 V}{4 a^{2}} .
$$

This can be rewritten as

$$
\begin{gathered}
\int K^{2} d V+2 \pi \Delta M=-\left.4 \pi a \phi \sin r\left(\phi^{\prime} \sin r+\phi \cos r\right)\right|_{R_{0}}+ \\
\left.2 \pi a \phi^{2} \sin r \cos r\right|_{R_{0}}+\pi a \int_{0}^{R_{0}} I d r-\frac{3 V}{4 a^{2}}
\end{gathered}
$$

where I is the same integrand as in Eq. (31). The first term is negative (on assuming (33)), and the integral is bounded above (from (34)) by $2 \pi L$. Thus we have

$$
\int K^{2} d V+2 \pi \Delta M<\left.2 \pi a \phi^{2} \sin r \cos r\right|_{R_{0}}+2 \pi L-\frac{3 V}{4 a^{2}} .
$$

$a \phi^{2} \sin r=\hat{R}$ is the natural areal coordinate, the equivalent of the Schwarzschild coordinate. Therefore we get

$$
\Delta M<\hat{R}_{0} \cos R_{0}+L-\frac{3 V}{8 \pi a^{2}}-\frac{1}{24 \pi} \int K^{2} d V
$$

Hence the mass of the inhomogeneity inside any given sphere cannot exceed the sum of the proper radius and the areal radius. This is a result that supports Einstein's view that "matter cannot be concentrated arbitrarily" [6]. 


\section{Nonhomogeneous cosmologies.}

In Sections IV, V and VI we have derived necessary and sufficient conditions for the appearance of trapped surfaces in spherical cosmologies. The only assumptions we make are contained in equation (33). This section, and the following ones will be devoted to showing that these conditions can be derived as a consequence of the Hamiltonian constraint (20), assuming that the mass excess be positive and localized.

We can conceive of two very different situations in which spherical symmetry could be assumed in a cosmological context. The local situation would be where the spherical symmetry holds only on a (small) patch of the data, a spherical galaxy in a universe with many such objects. We will discuss this case first. The other case is when the spherical symmetry holds globally, we have only one spherical lump in the whole universe. We postpone discussion of this situation until Section IX.

In the last few sections we were concerned with universes containing an isolated spherically symmetric lump. There may be a large number of such bumps, some of them with excess energy but many with deficit energy. Direct integration of the Lichnerowicz equation (Eq. (20)) shows that the total energy of all bumps vanishes. If the universe on average is homogeneous and isotropic we expect that there exist regions with positive and negative excess energy.

We assume that those regions are separated so that in between them the metric approaches the Friedmann-Lemaitre-Walker metric, i.e., the conformal factor $\phi$ tends to 1 outside the perturbed region and the extrinsic curvature vanishes (except, of course, for the Hubble constant term). We place the north pole at the center of the given lump. 
Then one might be more specific and say that $\phi$ tends to 1 before reaching the coordinate distance $r=\pi / 4$ or even $r=5 \pi / 12$. That is not a severe restriction since, as pointed above, there might exist many bumps and a zone of influence of any of these may cover only a small fraction of the whole universe.

Keeping this in mind, we prove the following:

Lemma 5. Assume that we are given an $r_{0}, 0<r_{0}<5 \pi / 12$ such that $\Delta \rho$ is nonnegative for $r \in\left(0, r_{0}\right)$ and that $\phi\left(r_{0}\right) \geq 1$ then

$$
\phi \geq 1, \quad \phi^{\prime} \leq 0 \quad \forall r \in\left(0, r_{0}\right)
$$

Proof. Using spherical symmetry, we may write equation (20) as follows:

$$
\nabla^{2} \phi=\frac{1}{a^{2} \sin ^{2} r}\left(\sin ^{2} r \phi^{\prime}\right)^{\prime}=-2 \pi \Delta \rho \phi^{5}-K^{2} / 12 \phi^{5}+3 /\left(4 a^{2}\right) \phi\left(1-\phi^{4}\right) .
$$

One easily checks that

$$
\alpha_{0}=\sup \left[3 /(4) \phi\left(1-\phi^{4}\right): 0 \leq \phi \leq 1\right]=0.6 / 5^{1 / 4}=0.4012442 .
$$

We will need below an equation related to (66),

$$
\nabla^{2} \chi=\frac{1}{a^{2} \sin ^{2} r}\left(\sin ^{2} r \chi^{\prime}\right)^{\prime}=\alpha_{0}
$$

Assume the contrary to the claim: let there exists a solution $\phi$ of $(24)$ such that $\phi^{\prime} \geq 0$ on an interval $\left(R_{i}^{*}, R_{i}\right)$. Let us remark that this means that $\phi<1$ in a part of this interval because if $\phi>1$ on the whole interval, then the right-hand-side of (66) is nonpositive. The maximum principle guarantees that $\phi$ cannot have an interior minimum, which contradicts the positivity of $\phi^{\prime}$. 
Assume that $\phi\left(R_{i}^{*}\right)=\chi\left(R_{i}^{*}\right)$; by definition $d / d r \phi\left(R_{i}^{*}\right)=0$. The equation (67) is solved by

$$
\chi=-\left(\alpha_{0} / 2\right) r \cot r+C_{i} .
$$

One can find that $\chi$ is increasing everywhere, so that at the point $R_{i}^{*}$ we have $d / d r \chi>$ $d / d r \phi$. Under the above stated initial conditions, and recognising that $\nabla^{2}(\chi-\phi)>0$, one may conclude that everywhere on the chosen interval

$$
d \phi / d r \leq d \chi / d r, \quad \phi \leq \chi .
$$

The change $\delta \phi=\phi\left(R_{i}\right)-\phi\left(R_{i}^{*}\right)$ of $\phi$ on $\left[R_{i}^{*}, R_{i}\right]$ is estimated from above by the change of $\chi$, given by $\chi(R)-\chi\left(R_{i}^{*}\right)$ (note that both functions are equal at $\left.R_{i}^{*} !\right)$. But the change of $\chi$ on all intervals $\bigcup_{1}^{n}\left(R_{i}^{*}, R_{i}\right), n \leq \infty$ on which $\phi$ is increasing is smaller than the change of $\chi$ on the entire interval $(0,5 \pi / 12)$, which is not greater than $\alpha / 3$. Since at that point $\phi \geq 1$, it means that $\phi \geq 1-\alpha_{0} / 3$.

Now, let us observe that the last term on the right hand side of (24) is decreasing for $\phi>1-\alpha_{0} / 3$. Define

$$
\alpha_{1}=\sup \left[3 \phi\left(1-\phi^{4}\right) / 4: 1 \geq \phi \geq 1-\alpha_{0} / 3\right],
$$

according to the above remark, $\alpha_{1}<\alpha_{0}$. Repeating the above described procedure, we will obtain a better estimation from below for the function $\phi$,

$$
\phi>1-\frac{\alpha_{1}}{3} .
$$

Repeating the above procedure infinitely many times, we finally arrive at the desired estimation

$$
\phi \geq 1 .
$$


From Eq. (69) we see that $\phi$ is monotonically decreasing, since the right hand side of (69) is nonpositive and we may apply the maximum principle. That ends the proof of the lemma.

Remark. That iteration procedure that has been described above works until one meets a fixed point of the map:

$$
\phi_{i+1}=1-\phi_{i}\left(1-\phi_{i}^{4}\right) / 4,
$$

i. e., a value $\phi_{*}$ such that

$$
\phi_{*}=1-\phi\left(1-\phi_{*}^{4}\right) / 4 .
$$

The only solution of the last equation is $\phi_{*}=1$.

One situation that is of interest is where one has an isolated lump. This is the situation where the deviation from the Friedmann background is localized in the sense that the conformal factor equals 1 somewhere in a region outside the lump. Such an isolated lump may be formed by having a region of enhanced density surrounded by a region with diminished density In such a situation we can prove

Lemma 6: Given three radii $r_{0}, r_{1}, r_{2}$ with $r_{0}<r_{1}<r_{2}, r_{0}<5 \pi / 12$ and $r_{1}<\pi / 2$, such that $\Delta \rho \geq 0$ for $r \in\left(0, r_{0}\right), \Delta \rho \leq 0$ for $r \in\left(r_{0}, r_{1}\right)$ and $\Delta \rho=0$ for $r \in\left(r_{1}, r_{2}\right)$. If $\phi=1$ for a particular $r \in\left(r_{1}, r_{2}\right)$ and if $\phi<(\sin r)^{-1 / 2}$ then $\phi>1, \phi^{\prime} \leq 0$ for $r \in\left(0, r_{1}\right)$.

We will postpone the proof of this Lemma until the end of Section X.

Lemma 6 and Lemma 3 can now be combined to prove

Theorem II: Given an isolated spherical lump with a central region of positive excess 
mass and if in that region we find a surface satisfying

$$
\Delta M-\Delta J>\frac{7}{6} L+H L^{2}-\frac{3 V}{8 \pi a^{2}}
$$

we must have a trapped surface.

We will also prove the following.

Lemma 7.If $\Delta \rho$ is nonnegative and if $\left.\left(\phi^{\prime} \sin r+\phi \cos r\right)\right|_{r_{0}} \geq 0$ then

$$
\phi^{\prime} \sin r+\phi \cos r \geq 0, \forall r \in\left(0, r_{0}\right) \text {. }
$$

Proof. Notice that

$$
\begin{gathered}
\frac{1}{a^{2} \sin r}\left(\phi^{\prime} \sin r+\phi \cos r\right)^{\prime}=\frac{1}{a^{2} \sin ^{2} r}\left(\sin ^{2} r \phi^{\prime}\right)^{\prime}-\phi / a \\
=-2 \pi \Delta \rho \phi^{5}-\left(\frac{\phi}{4 a^{2}}+3 \frac{\phi^{5}}{4 a^{2}}\right)-K^{2} \phi^{5} / 12
\end{gathered}
$$

The last equation follows from the constraint equation (24). The quantity $\phi^{\prime} \sin r+\phi \cos r$ is decreasing; but at $r_{0}$ it is $>0$, hence $\phi^{\prime} \sin r+\phi \cos r$ is positive everywhere for $r \in\left(0, r_{0}\right)$.

\section{Spherically symmetric constant scalar curvature manifolds}

Until now we have been dealing with localized spherically symmetric lumps which we regarded as being embedded in a Friedmann background. The class of spherically symmetric cosmologies contains many other solutions. In particular, we wish to investigate solutions which are globally spherically symmetric. The general situation we wish to consider consists of a spherically symmetric initia l data set which has a lump of excess matter surrounded by a constant density background which fills the rest of the universe. 
Outside the lump, the Hamiltonian constraint guarantees that the scalar curvature is constant. The combination of spherical symmetry and constancy of the scalar curvature guarantees that that section of the manifold can be regarded as part of a standard round $S^{3}[7]$. This is not equivalent to guaranteeing that the conformal factor must be identically one in the exterior region. We need to understand the conformal structure of spherically symmetric constant scalar curvature manifolds.

Let us return to the Hamiltonian constraint, Eq. (19)

$$
\nabla^{2} \phi=\frac{3}{4 a^{2}}\left(\phi-\phi^{5}\right)-\left[\frac{1}{12} K^{2}+2 \pi\left(\rho-\rho_{0}\right)\right] \phi^{5},
$$

and let us consider the simplest possible case. Let us assume that the matter is at rest, which gives us $\mathrm{K}=0$, and that the energy density is a constant, $\rho=\rho_{0}$. The Hamiltonian constraint now simplifies enormously, to give

$$
\nabla^{2} \phi=\frac{3}{4 a^{2}}\left(\phi-\phi^{5}\right)
$$

Obviously, one solution of (76) is $\phi \equiv 1$, which is the standard solution. This is not the only one. There is a complete family of regular solutions of Eq. (76) given by

$$
\phi_{(\alpha)}=\frac{\alpha^{\frac{1}{2}}}{\left(\alpha^{2} \cos ^{2} \frac{r}{2}+\sin ^{2} \frac{r}{2}\right)^{\frac{1}{2}}}
$$

where $\alpha$ is an arbitrary positive constant.

In Eq. (76) we are trying to find a conformal factor $\phi$ which transforms a manifold with constant scalar curvature ${ }^{(3)} R=6 / a^{2}$ to another manifold with the same constant curvature ${ }^{(3)} R=6 / a^{2}$. The Yamabe Theorem [8] states that every Riemannian manifold can be conformally transformed into on e with constant scalar curvature. The existence 
part of the Yamabe theorem has bee $\mathrm{n}$ shown to be true. Whether the resulting constant scalar curvature manifold is unique is an open problem. However, it is known that in the case of flat space we do not have uniqueness; there is at least a one parameter family of inequivalent manifolds. The conformal factors mapping between them are just the $\phi_{(\alpha)}$ 's given by Eq. (77). The manifold with metric

$$
d S^{2}=\phi_{(\alpha)}^{4} a^{2}\left[d r^{2}+\sin ^{2} r d \Omega^{2}\right]
$$

can be shown to be a round metric by using the coordinate transformation [7]

$$
L=\pi-2 \tan ^{-1}\left(\alpha \cot \frac{r}{2}\right) .
$$

It is important that we understand the general structure of the $\phi_{(\alpha)}$ 's. It is clear from (77) that $\phi_{(\alpha)}$ with $\alpha=1$ satisfies $\phi \equiv 1$ and so represents the identity transformation. Each $\phi_{(\alpha)}$, with $\alpha \neq \equiv 1$, equals $1 / \sqrt{\alpha}$ at $\mathrm{r}=0$; equals $\left[2 \alpha /\left(\alpha^{2}+1\right)\right]^{1 / 2}$, which is always less than 1 , at $r=\pi / 2$; and equals $\sqrt{\alpha}$ at $r=\pi$. If $\alpha<1$ then $\phi_{(\alpha)}$ monotonically decreases, if $\alpha>1, \phi_{(\alpha)}$ monotonically increases. The value of $\mathrm{r}$, call it $R_{1}$, at which each $\phi_{(\alpha)}$ passes through 1 is given by

$$
\alpha=\tan ^{2} \frac{R_{1}}{2} .
$$

In the range $0 \leq r \leq \pi / 2$ the $\phi_{(\alpha)}$ 's with $\alpha>1$ give a smooth set of non-intersecting curves with one curve passing through each point in the $(r, \phi)$ plane with $\phi_{(\alpha)}$ in the range $0<\phi<1$. At each fixed value of $\mathrm{r}$ as $\alpha$ increases, $\phi$ decreases. Each individual curve is increasing as $\mathrm{r}$ increases but they are all still less than 1 on reaching $r=\pi / 2$. Beyond $r=\pi / 2$ each curve in turn crosses the $\phi=1$ line at increasing values of $R_{1}$ with increasing $\alpha$ (as given by (80)) without intersecting any other curve. However, each $\phi_{(\alpha)}$ is climbing 
more rapidly than the curves with lower values of $\alpha$ by the time it crosss the line $\phi=1$. Above this line the curves proceed to cross because a curve with higher values of $\alpha$ must rise above all the curves with lower $\alpha$ by the time it reaches $r=\pi$.

Superimposed on this pattern is a mirror-image set of curves for values of $\alpha<1$. These start with values greater than 1 at $\mathrm{r}=0$, the smaller $\alpha$ the greater the value. They then proceed to decrease as $\mathrm{r}$ increases, crossing one another as the curves with smaller values of $\alpha$ fall below the curves with larger values of $\alpha$. All the crossing is accomplished by the time they cross the $\phi=1$ line, and this all happens before one reaches $r=\pi / 2$. Beyond $r=\pi / 2$ all the curves smoothly decrease without crossing until one reaches $r=\pi$. The part of $(\phi, r)$ space defined by $\phi^{2} \sin r>1$ contains no curves. Through every other point two curves, with different values of $\alpha$, pass.

Now let us consider one of these constant scalar curvature manifolds, with line-element given by (75). When we substitute the explicit form of $\phi_{(\alpha)}$ into Eq. (21) we get

$$
\nabla_{a} n^{a}=2(a \alpha \sin r)^{-1}\left[\alpha^{2} \cos ^{2}(r / 2)-\sin ^{2}(r / 2)\right]
$$

This quantity has only one zero at $r=R_{E}$ given by

$$
\alpha=\tan \frac{R_{E}}{2}
$$

An alternative form is

$$
\cos R_{E}=\frac{1-\alpha^{2}}{1+\alpha^{2}}
$$

This value $\left(R_{E}\right)$, from $(79)$, corresponds to $L=\pi / 2$, i.e., the physical equator of the conformally transformed space. $\nabla_{a} n^{a}$ is positive for all $r<R_{E}$ and is increasingly negative for $r>R_{E}$. Therefore, independent of the value of $\alpha$, all the manifolds share the 
property of the standard Friedmann slice that they have no trapped surfaces in the "northern' hemisphere, but that as one approaches the 'south' pole all the two-spheres become trapped.

The equator, where $\alpha=\tan \frac{R_{E}}{2}$, also plays another role which will be important in our future discussions. Consider one of these curves $\phi_{(\alpha)}$, with a given $\alpha$, and let us assume $\alpha>1$. Start on this curve at $\mathrm{r}=0$, with $\phi=1 / \sqrt{\alpha}<1$. As one moves along this given curve, other $\phi_{(\alpha)}$ 's, with different values of $\alpha$, cross it. These curves will have $\alpha<1$. These begin with curves whose value of $\alpha$ is essentially zero. As $r$ increases, as we move along our chosen curve, the value of $\alpha$ of the curves crossing it monotonically increases, but is still less than 1 as we pass $r=\pi / 2$. Finally, at $R_{1}$, given by Eq. (80), with $R_{1}>\pi / 2$, the curv e crosses the line $\phi=1$ (which is, of course, the $\phi_{(\alpha)}$ with $\alpha=1$ ). The chosen curve now starts to overtake curves with values of $\alpha>1$ but less than our chosen $\alpha$. This continues until one reaches the the equator, $r=R_{E}$, given by eqns.(79) and (80). Note $R_{E}>R_{1}$ for $\alpha>1$. This point also satisfies $\phi^{2} \sin R_{E}=1$ so is the boundary of the excluded region. Only one curve, our chosen one, passes through this point. From this point on, as we move along our curve in a direction of increasing $r$, our chosen curve is now being overtaken by curves $\phi_{(\alpha)}$ with larger and larger $\alpha$ 's. Just as the vertical line $r=0$ is essentially the curve $\phi_{\alpha}$ with $\alpha=0$, the vertical line $r=\pi$ is the $\phi_{\alpha}$ with $\alpha=\infty$.

\section{Spherically symmetric models with non-constant scalar curvature}

We wish to construct a manifold which is spherically symmetric with a given (nonconstant) scalar curvature R. We can assume that the metric is of the form (9), and then 
we can write the equation satisfied by $\phi$ in a form very similar to equation (75), i.e.,

$$
\nabla^{2} \phi=\frac{3}{4 a^{2}}\left(\phi-\phi^{5}\right)-\frac{1}{8} \Delta R \phi^{5},
$$

where $\Delta R=R-6 / a^{2}$.

As we mentioned earlier, we wish to consider manifolds with constant density outside some compact region. Thus we will wish to consider situations where $\Delta R$ is zero outside some finite spherical volume. In the exterior region the equation for $\phi$ reduces to

$$
\nabla^{2} \phi=\frac{3}{4 a^{2}}\left(\phi-\phi^{5}\right) .
$$

We know a complete family of solutions to this equation, the $\phi_{(\alpha)}$ 's of Eq. (77). However, it is not immediately obvious that the solution of Eq. (85), which is only valid on part of the manifold, must be one of these $\phi_{(\alpha)}$ 's. In one case we need regularity both at $r=0$ and at $r=\pi$. In the other case we need regularity only at, say, $r=\pi$. We know that any solution to (84) must have some finite positive value at $r=\pi$, and that it's first derivative must vanish there. We have a $\phi_{(\alpha)}$ with the same value at $r=\pi$ and with the same first derivative. This would be usually enough to prove that the two functions must coincide. We cannot immediately deduce this here because the point $r=\pi$ is a singular point of the equation because $\sin r=0$ there. Happily, Eq. (85) i s really only an ordinary differential equation because of the spherical symmetry and a treatment exists of such singular points in Rendall and Schmidt [9]. Using their Theorem 1 allow us to deduce that the two solutions must coincide and that the solution of Eq. (85) must be one of the $\phi_{(\alpha)}$ 's in the exterior of the support of $\Delta R$.

One obvious situation we would like to consider is when there is a step-function in the scalar curvature, when the scalar curvature has one (constant) value in part of the 
manifold and has a different (constant) value on the rest. It is easy to see that the conformal factor to achieve this must made up of a pair of the functions $\phi_{(\alpha)}$ defined by Eq. (77). More precisely, we would want that the conformal factor equal $C_{1} \phi_{\left(\alpha_{1}\right)}$ in one region and $C_{2} \phi_{\left(\alpha_{2}\right)}$ in the rest, where $C_{1}$ and $C_{2}$ are prescribed constants depending on the values of the scalar curvature and $\alpha_{1}$ and $\alpha_{2}$ are adjustable parameters. One now tries to match these functions and their first derivatives at some radius and discovers that this can not be done, irrespective of the values of $C_{1}, C_{2}$ or the coordinate value of the matching point..

This means that we cannot expect to be able to solve Eq. (84) with a randomly chosen $\Delta R$. Equally, if we get a solution it may well not be unique. Nevertheless, we have been able to extract a number of interesting and useful properties of solutions to Eq. (84) (assuming one exists!). The situation we are interested in is when $\Delta R \geq 0$ and has compact support. In the exterior region we know that the solution must be one of the $\phi_{(\alpha)}$ 's. We will show that this $\alpha$ must satisfy $\alpha<1$ if the support of $\Delta R$ is not too large.

The assumption we will make is that the support of $\Delta R$ lies entirely in the 'northern' hemisphere. We do not define this in terms of the background geometry, but rather in terms of the physical metric. The exterior solution is defined by a $\phi_{(\alpha)}$ with $\alpha=\alpha_{0}$. We assume that the 'equator', the radius $R_{E}$ satisfying

$$
\alpha_{0}=\tan R_{E} / 2
$$

lies in the exterior zone.

The analysis is based entirely on the maximum principle as applied to Eq. (84). The solution $\phi$ to (84) cuts through a complete family of $\phi_{(\alpha)}$ 's. At any point along $\phi$, we know 
that the two $\phi_{(\alpha)}$ 's that pass through that point satisfy

$$
\nabla^{2} \phi_{(\alpha)}=\frac{3}{4 a^{2}}\left(\phi_{(\alpha)}-\phi_{(\alpha)}^{5}\right)
$$

Therefore we must have at that point since we assume $\Delta R \geq 0$

$$
\nabla^{2}(\phi(\alpha)-\phi) \geq 0
$$

If $\Delta R>0$ at this point then by continuity $\nabla^{2}\left(\phi_{(\alpha)}-\phi\right)>0$ close to this point. This implies, due to the maximum principle, that if $\phi$ approaches a $\phi_{(\alpha)}$ from above it must pass through it; it cannot either 'bounce' off it or merge with it.

We obtain our result by contradiction, so we begin by assuming that $\alpha_{0}>1$. Let us start at $r=\pi$ and move along the curve $\phi$, the solution to (84). In this region it coincides with $\phi_{\alpha_{0}}$. We continue along this curve through $R_{E}$ (as defined by (85)). At some radius $r<R_{E}$ we enter the support of $\Delta R$. At this point the curve $\phi$ must bend downwards, away from $\phi_{\alpha_{0}}$. But in this region the space underneath $\phi_{\alpha_{0}}$ is filled with all the curves $\phi_{(\alpha)}$ with $\alpha>\alpha_{0}$. Our solution curve must continue descending faster than these $\phi_{(\alpha)}$ 's. As we point out above, it can neither merge with, nor bounce off, any of them. This can only lead to disaster. Either $\phi$ goes to zero, or hits the $r=0$ point with a non-zero angle (if $\phi^{\prime}=0$ at $\mathrm{r}=0$, it would be tangent to one of the $\phi_{(\alpha)}$ 's). Thus the original assumption, that $\alpha_{0}>1$ cannot be true. A similar disaster befalls a curve which starts with $\alpha_{0}=1$. Therefore we must conclude that $\alpha_{0}<1$.

Each point of $(r, \phi)$ space (excepting the region defined by $\phi^{2} \sin r \geq 1$ ) has two curves $\phi_{(\alpha)}$ passing through it. Thus any curve $\phi(r)$ can be described by giving the pair of $\alpha$ values corresponding to each point it passes through. Consider the particular curve we describe 
in the preceeding paragraph. As it moves away from $r=\pi$ one of the $\alpha$ 's remains fixed at $\alpha_{0}$, the other starts at $\infty$ and monotonically decreases, at $R_{E}$ both $\alpha$ 's coincide, and at values of $r<R_{E}$ the varying $\alpha$ is less than $\alpha_{0}$. Now we enter the support of $\Delta R$. The curve $\phi(r)$ now drops below the curve $\phi_{\left(\alpha_{0}\right)}$. One $\alpha$ continues to decrease, the other increases above $\alpha_{0}$. The key question is what happens to this increasing $\alpha$ ? It cannot reach a maximum value either at or before reaching $r=0$, because this is equivalent to being a tangent (from above) to one of the $\phi_{(\alpha)}$ 's, which is forbidden by the maximum principle. Thus something bad must happen to the curve in question, it either goes to zero at a nonzero value of $r$, or $\phi^{\prime}>0$ at $r=0$.

We can now deduce somewhat more assuming, as always, that $\Delta R \geq 0$ and that it has support only on one side of the equator. We now assume $\alpha_{0}<1$. Let us follow the solution to (84) in from $r=\pi$. We start off at a value of $\phi=\left(\alpha_{0}\right)^{1 / 2}<1$ and it monotonically increases as we move along $\phi_{\alpha_{0}}$. We will not deviate from this curve until we, at least, reach the equator $\left(R_{E}\right)$. By this point $\phi_{\alpha_{0}}$ has risen above 1 . The solution $\phi$ starts curling downwards relative to $\phi_{\alpha_{0}}$ at some $r<R_{E}$. However, it cannot curl down too much, because if it passes, going downwards, through $\phi=1$ it is stuck in the same trap as before. We therefore can assume that $\phi>1$ in the interior. But, as long as $\phi$ is $>1$, we have $\nabla^{2} \phi<0$ and this means that $\phi$ cannot have a minimum in the support of $\Delta R$. Hence we must have $\phi^{\prime} \leq 0$ in the interior, with equality only at $\mathrm{r}=0$. Of course, since $\alpha_{0}<0$ we have $\phi^{\prime}<0$ in the exterior.

The result that the conformal factor outside the support of the excess matter must coincide with one of the special functions $\phi_{(\alpha)}$ is interesting insofar as it shows that the 
exterior region is homogeneous and isotropic, uninfluenced by the lump. This is exactly analogous with the result derived in Section II that the current potential K vanishes outside the support of the current.

Let us now return to the promised proof of Lemma 6 from Section VIII. Here we consider a situation where part of the manifold consists of a region $\left(0, r_{0}\right)$ near the north pole with $\Delta R \geq 0$, a second region $\left(r_{0}, r_{1}\right)$ with $\Delta R \leq 0$ and that we have standard FLW data outside these regions. This means that the conformal factor, the solution to (20), equals 1 outside $r_{1}$. If we follow the conformal factor $\phi$ in from the right it must increase as it enters the region of negative $\Delta \rho$. If it went down there would be an interval $\left(r_{1}-\delta, r_{1}+\delta^{\prime}\right)$ in which $\phi \leq 1$. But we have from (83), since $\Delta R \leq 0$,

$$
\nabla^{2} \phi \geq 0
$$

However $\phi$ achieves an interior maximum at $r=r_{1}$, which is forbidden. Therefore we must have $\phi \geq 1$ as we approach $r_{1}$.

Having shown that $\phi>1, \phi^{\prime}<0$ near $r_{1}$, we now wish to show that $\phi>1$ in the whole interval $\left(r_{0}, r_{1}\right)$. Let us assume that $\phi^{\prime}=0$ somewhere in the interval $\left(r_{0}, r_{1}\right)$. If $\phi^{2} \sin r<1$ in the interval, this means that $\phi$ must turn over in the region that is filled with $\phi_{\alpha}$ curves. In particular, this means that the $\phi$ curve must tangent from below one of these curves, say $\phi_{\alpha_{0}}$, at some point, say $r=r_{3}$. At this point we have

$$
\phi\left(r_{3}\right)=\phi_{\alpha_{0}}\left(r_{3}\right)>1, \quad \phi^{\prime}\left(r_{3}\right)=\phi_{\alpha_{0}}\left(r_{3}\right)<0 .
$$

In a neighbourhood of $r_{3}$ we also have

$$
\phi \leq \phi_{\alpha_{0}} .
$$


Subtracting (87) from (84) gives

$$
\nabla^{2}\left(\phi_{\alpha_{0}}-\phi\right)=\frac{3}{4 a^{2}}\left[\phi_{\alpha_{0}}-\phi_{\alpha_{0}}^{5}-\phi+\phi^{5}\right]+\frac{1}{8} \Delta R \phi^{5} .
$$

The right-hand-side of (88) is non-positive, so the minimum principle says that $\phi_{\alpha_{0}}-\phi$ cannot achieve a minimum at $r_{3}$, which contradicts our assumptions. Hence $\phi^{\prime} \leq 0$ on the whole interval $\left(r_{0}, r_{1}\right)$, with equality only in the FLW region. Therefore $\phi\left(r_{0}\right)>1$. Now we can use Lemma 5 to finally show $\phi>1, \phi^{\prime}<0$ in the interval $\left(0, r_{0}\right)$.

\section{Trapped surfaces in spherical universes.}

In the previous section we have proven that a spherical bump of compact support confined to the northern hemisphere produces a monotonically decreasing conformal factor $\phi$,

$$
\phi^{\prime} \leq 0,
$$

if its excess energy is non-negative. This corresponds to Lemma 5 of Sec. VIII. We can also prove

$$
\phi^{\prime} \sin r+\phi \cos r \geq 0
$$

Outside the bump $\phi$ must coincide with $\phi_{\alpha}$, where $\alpha<1$. As is shown in Lemma 7, the quantity $\phi^{\prime} \sin r+\phi \cos r$ monotonically decreases. Outside the matter it is equal to

$$
\alpha^{-1} \phi^{3}\left[\left(1+\cos ^{2} r\right)\left(\alpha^{2}-1\right)+2 \cos r\left(1+\alpha^{2}\right)\right] .
$$

This expression vanishes at a value $R_{1}$ such that $\cos R_{1}=(1-\alpha) /(1+\alpha)$, (Eq. (79)),i.e., when $\phi_{\alpha}$ crosses through 1 . Therefore inside a region with non-negative matter density the inequality (73) holds. 
Thus we can prove:

Theorem III: Given a globally spherically symmetric initial data set which satisfies $\operatorname{trK}=$ constant with a localized positive lump which is instantaneously at rest. If we can find a spherical surface satisfying

$$
\Delta M>L+\frac{A H}{4 \pi}-\frac{3 V}{8 \pi a^{2}}
$$

then that surface is trapped.

We can also prove:

Theorem IV: Given a globally spherically symmetric initial data set which satisfies $\operatorname{trK}=$ constant with a localized positive lump may be moving. If we can find a spherical surface satisfying

$$
\Delta M-\Delta J>\frac{7}{6} L+\frac{A H}{4 \pi}-\frac{3 V}{8 \pi a^{2}}
$$

then that surface is trapped.

In addition, one can prove another version of a sufficient condition (Lemma 3) for the formation of trapped surfaces by moving inhomogeneities. Indeed, let us return to the equation (68). In section VII we estimated $\Gamma$ from above by $H L^{2}$. The other estimate is that $\Gamma<8 \mathrm{Ha}^{2}$. This is obtained when one realises that $\phi$ merges with some $\phi_{\alpha}$, with $\alpha<1$ in the exterior, and that it must lie below this $\phi_{\alpha}$ in the interior. In turn, we know $\phi_{\alpha}<\alpha^{-1 / 2}$. Thus, if we replace $\phi^{4}$ in (82) with $\alpha^{-2}$ we will get an upper bound

$$
\Gamma<4 H a^{2} \alpha^{-2} \int_{0}^{R} \sin r \cos r d r=2 H a^{2} \alpha^{-2} \sin ^{2} R .
$$

This, as it stands is not a particularly interesting inequality until one realises that we are assuming that the excess matter is confined within the equator and that we are not 
interested in finding trapped surfaces beyond the equator. Therefore, we can replace $R$ in (92) by $R_{E}$ as given by (83). It is a straightforward manipulation of trigonometric functions to show

$$
\alpha^{-2} \sin ^{2} R_{E}=\frac{4}{\left(1+\alpha^{2}\right)^{2}}<4,
$$

which gives the desired inequality. Using (89) and proceeding as in Section VI, we obtain the following version of Lemma 3:

Theorem V: Under the preceeding conditions, if we obtain a surface for which

$$
\Delta M-\Delta J>\frac{7}{6} L+\frac{2 a^{2} H}{\pi}-\frac{3 V}{8 \pi a^{2}},
$$

either the surface itself is trapped or the interior contains trapped surfaces.

\section{Concluding remarks.}

Let us summarize the whole discussion. In this paper we study spherically symmetric closed universes. There are two different situations which are of interest.

First, we may have a homogeneous and spherically symmetric background geometry with many spherical bumps placed in it. Globally, a resulting universe is neither spherically symmetric nor homogeneous, but locally, close to a particular lump, there is a spherical symmetry with respect to the centre of the lump. Also, very far from the bump, the geometry of the chosen Cauchy slice coincides with the homogeneous and spherically background geometry. That case was studied in Section VIII. In Section VIII is formulated a sufficient condition (Theorem II) for the formation of trapped surfaces by moving perturbations, when the initial momentum of the gravitational field is changed. Obviously, Theorem II can be specialized to the case where the excess matter is at rest. Theorem I in Section V 
gives us a nice necessary condition for the formation of a trapped surface when the matter is at rest. Interestingly, we have failed to find an equivalent necessary condition in the case where the matter is moving. In Section VII we prove that in a sphere of a fixed radius only a finite amount of perturbed energy can be packed.

Secondly, one might be interested in investigating geometry of a universe that is not homogeneous but is globally spherically symmetric. Section IX contains a description of spherically symmetric constant scalar curvature models. These solutions are used in Section $\mathrm{X}$ to derive a number of properties of initial data for nonhomogeneous distributions of matter. Using them, we prove in Sec. XI criteria for the formation of trapped surfaces in globally spherically symmetric geometries that are generated by a single bump confined to only one hemisphere.

We would like to stress that these results obtained here are correct in the full nonlinear theory, nowhere do we draw on a perturbation expansion or a linear approximation. We expect that these results are true also for a class of nonspherical perturbations, for instance in the class of conformally flat perturbations of the homogeneous closed universes we would expect to obtain results analogous to those known in open flat universes [10].

Acknowledgements. We would like to thank the Physics Department, University College Cork for inviting one (ME) of us to Cork, where part of this work was done. N. Ó M. would like to thank the members of the Max Planck Institut für Astrophysik in Garching, especially Alan Rendall and Uwe Brauer, for their help and interest. N. Ó M. would also like to thank Jemal Guven. This work has been supported by the Polish Government Grant no. PB 2526/2/92. 


\section{References.}

[1] R. Penrose, Phys. Rev. Lett. 14, 57 (1965); Techniques of Differential Topology in Relativity (Soc. Ind. Appl. Math. 1972).

[2] S. W. Hawking, G.F.R. Ellis, The large scale structure of space-time (Cambridge University Press, Cambridge 1973).

[3] C. W. Misner, K. Thorne, J. Wheeler, Gravitation (Freeman, San Francisco 1973).

[4] P. Bizoń, E. Malec, N.Ó Murchadha, Phys. Rev. Lett. 61, 1147(1988); Class. Quantum Grav. 6, 961 (1989); Class. Quantum Grav. 7, 1953 (1990).

[5] U. Brauer, E. Malec, Phys. Rev. D45, R1836 (1992).

[6] A. Einstein Ann. Math. 40, 922 (1939).

[7] J. Guven and N. Ó Murchadha, to be published.

[8] R. Schoen, J. Diff. Geom. 20, 479 (1984).

[9] A. Rendall, B. Schmidt, Class. Quantum Grav. 8, 985 (1991).

[10] P. Koc, E. Malec, Acta Phys. Pol.B23, 123 (1992). 